A N N A L E S Annales de Bretagne et des Pays de l'Ouest

Anjou. Maine. Poitou-Charente. Touraine

111-4 | 2004

Varia

\title{
Réflexions sur la bretonnité
}

\section{Pierre-Jean Simon}

\section{OpenEdition}

\section{Journals}

Édition électronique

URL : http://journals.openedition.org/abpo/1188

DOI : $10.4000 / a b p o .1188$

ISBN : 978-2-7535-1496-6

ISSN : 2108-6443

Éditeur

Presses universitaires de Rennes

Édition imprimée

Date de publication : 20 décembre 2004

Pagination : 141-149

ISBN : 978-2-7535-0082-2

ISSN : 0399-0826

Référence électronique

Pierre-Jean Simon, «Réflexions sur la bretonnité », Annales de Bretagne et des Pays de l'Ouest [En ligne], 111-4 | 2004, mis en ligne le 20 décembre 2006, consulté le 19 avril 2019. URL : http:// journals.openedition.org/abpo/1188; DOI : 10.4000/abpo.1188 


\title{
Conclusion : Réflexions sur la bretonnité
}

\author{
Pierre-Jean SIMON \\ Professeur honoraire de sociologie \\ Université Rennes 2 Haute-Bretagne
}

Se donner comme objet d'étude la bretonnité - c'est-à-dire se poser les questions : comment est-on Breton? Qu'est-ce qui fait que les Bretons sont des Bretons, selon des manières fort diverses et aussi à différents degrés, à ses propres yeux et aux yeux des autres? Que recouvre et que signifie cette identité que certains d'entre eux revendiquent et qui leur est aujourd'hui plus volontiers qu'autrefois reconnue? - c'est, on le perçoit tout de suite, s'interroger sur une ethnicité pour le moins problématique. Mais, à vrai dire, malgré parfois certaines apparences, toutes les ethnicités, si l'on y regarde d'un peu près, sont, chacune à sa manière, problématiques. Et celle-ci, la bretonnité, ne l'est sans doute ni plus ni moins qu'une autre. De sorte que l'analyse que l'on en peut mener peut valoir pour bien d'autres. C'est, en tout cas, ce que j'ai essayé de faire : établir, à partir de la bretonnité, une grille de description, de compréhension, d'analyse des diverses ethnicités que l'on rencontre dans le monde contemporain en France, en Europe et ailleurs ${ }^{1}$.

Il convient, cependant, de faire ici trois remarques initiales. J'utilise pour désigner et décrire la bretonnité, le concept d'ethnicité. Ce mot même peut, on le sait, prêter à équivoque, ce qui est " ethnique " évoquant assez souvent dans le langage courant et celui des médias les idées de race, les traits physiques, l'origine biologique et sa transmission par le sang ou par les gènes - sans parler de la sinistre " purification ethnique ". Précisons donc, en y insistant très fortement, que l'on se situe ici dans une tradition de la sociologie où ces termes se trouvent dégagés de toute connotation raciale. Il doit être bien clair que l'ethnicité telle que j'en parle n'a rien, absolument rien à voir avec la race, l'atavisme, le pedigree, c'est-à-dire avec des conceptions vétérinaires appliquées aux êtres humains. L'ethnicité ce n'est absolument pas un fait de nature. C'est un fait de culture et d'histoire. Autrement dit d'héritage social et pas du tout d'hérédité biologique.

1. Simon, Pierre-Jean, La Bretonnité. Une ethnicité problématique, Rennes, Terre de Brume/Presses Universitaires de Rennes, coll. «Essais », 1990. 
Pour éviter les fâcheuses connotations dues à l'histoire du mot ethnique et de ses dérivés, et donc les interprétations intempestives auxquelles peut donner lieu le concept d'ethnicité, on pourrait, si c'était pratiquement possible (mais je ne pense pas que ce le soit), remplacer ce terme par celui d'Xéité, en utilisant alors un langage aussi proche que possible du langage formel des mathématiques, dans la mesure où il s'agit de comprendre et d'analyser comment un ensemble collectif $X$ (ce peut être les Bretons, les Siciliens, les Corses, les Basques, les Catalans, les Gallois, les Québécois, etc.) est ce qu'il est, comment ses membres, en certaines situations, se définissent eux-mêmes et sont définis par les autres, se reconnaissent et sont reconnus comme tels, par quoi un ensemble collectif $X$ se distingue des autres ensembles collectifs similaires.

On pourrait donc en ce sens parler - ce qui éviterait les querelles sur les mots, qui n'ont jamais grand intérêt - de différentes Xéités ou d'une Xéité particulière. Ainsi de l'Xéité bretonne ou bretonnité, de l'Xéité sicilienne ou sicilianité, de l'Xéité catalane ou catalanité, etc. L'ethnicité bretonne ou l'Xéité bretonne ce serait ainsi ce qui fait que les Bretons sont des Bretons, comme les Corses sont Corses, les Siciliens Siciliens, les Catalans Catalans, etc.

Pourquoi en deuxième lieu, parler d'ethnicité - ou, si l'on veut, d'Xéité plutôt que, comme on le fait assez généralement aujourd'hui, d'identité? Il me paraît préférable pour ma part, de ne parler d'identité que pour les aspects subjectifs de l'ethnicité De réserver, autrement dit, ce terme pour l'identification, le sentiment d'appartenance. L'ethnicité, en effet, l'ethnicité bretonne, par exemple, c'est l'identité sans doute, mais ce n'est pas seulement l'identité. L'ethnicité englobe l'identité, mais ne se confond pas avec elle. L'identité est une notion, il faut bien le reconnaître, assez floue et à géométrie variable. Ce qui, d'une certaine manière, est bien commode, car on peut y mettre à peu près tout ce que l'on veut. Parler d'identité cela permet souvent, en fait, de se situer seulement sur le plan des représentations, des opinions, des sentiments, des subjectivités, en faisant l'économie des interrogations plus avant sur ce qui, éventuellement, fonde ces représentations, sur ce qui est au fondement de cette identité et qui la justifie. L'identité culturelle ou régionale, ainsi l'identité bretonne, ce serait l'image de soi et de la collectivité à laquelle on appartient. L'image de soi comme différent des autres et semblable à certains autres. Image rassurante de différence partagée. Mais cette différence quelle est-elle? Une collectivité ce n'est pas seulement un sentiment d'appartenance et d'identification. Ce n'est pas uniquement une affaire de subjectivité. Et de subjectivité individuelle, d'individus juxtaposés et n'existant que par euxmêmes, qui pourraient à leur gré, selon des " stratégies " plus ou moins utilitaires, choisir leur identité, une identité personnelle. Laquelle, selon les conceptions individualisées de la culture qui, plus que jamais, prédominent aujourd'hui, pourrait beaucoup plus facilement être reconnue, dans nos sociétés d'individus, que les identités collectives, qui renvoient, elles, à ces notions beaucoup plus gênantes de groupes ethniques, de collecti- 
vités de culture et d'histoire, de minorités régionales ou autres - et dont la reconnaissance est d'ailleurs, pour ce qui est du cas de la France, toujours interdite par l'idéologie nationale-républicaine qui ne veut connaître, résolument opposée à tout ce qui pourrait ressembler à du " communautarisme ", que des individus : les individus-citoyens que sont les nationaux et les individus étrangers, les non-nationaux.

On ne peut ainsi pour l'identité bretonne éluder la question : appartenance et identification à quoi? Pour être Breton il ne suffit certainement pas de se dire Breton, de se sentir Breton, de s'affirmer et se revendiquer tel, d'écouter de la musique bretonne, ou, comme disait Per Jakez Hélias, de mettre un gwenn ha du sur sa voiture et de courir les festou noz. Il paraît bien difficile d'admettre que l'on serait ainsi plus Breton qu'un bretonnant au quotidien, que celui qui a conservé l'usage de la langue bretonne, paysan ou marin-pêcheur, mais qui n'aurait aucun sentiment breton particulier, pas d'identité bretonne affichée, s'identifiant en fait bien davantage - cela arrive - à la France qu'à la Bretagne. (On peut toujours dire, bien sûr, que ce bretonnant-là, sans identité, sans subjectivité bretonne, est aliéné, qu'il a été lobotomisé par les majoritaires, par l'enseignement du mépris de lui-même. Ce qui n'est pas faux. Mais il n'en est pas moins objectivement Breton.)

Il est vrai, certes, que l'on peut être Breton ou Bretonne de bien des façons. Y compris de cœur, comme on dit, sans avoir aucune origine bretonne. La Bretagne n'a pas de papiers, comme disait Morvan Lebesque, et il n'y a sans doute pas lieu de le regretter : les papiers d'identité, les passeports et les permis de séjour, c'est sûrement un signe de modernité, ce n'est pas forcément un signe de haute civilisation. Au contraire de ce qu'il en va pour la francité, il n'est formellement demandé à personne de faire la preuve de sa bretonnité par le droit du sang ou par le droit du sol. Mais, cela dit, il ne faut tout de même pas se leurrer et raconter n'importe quoi. Être Breton et se voir reconnu comme tel par les gens en Bretagne - car que serait une identité qui ne serait que pour soi-même, sans être reconnue par personne, sinon un fantasme? - c'est d'abord et avant tout une certaine manière d'être, d'exister au monde, de se comporter avec les autres, et entretenir un rapport particulier avec les choses et les gens de Bretagne.

Cela n'a rien, encore une fois, absolument rien à voir avec les liens du sang entendus avec des significations plus ou moins raciales, racialistes ou racistes, ces représentations dont il convient de complètement se débarrasser - encore que cela ne soit pas si facile, la bonne volonté ne suffit pas, car chassées par la porte il arrive qu'elles reviennent par la fenêtre, y compris celle de l'identité. Mais n'étant pas du tout une question d'hérédité biologique, la bretonnité a à voir, comme toute ethnicité, avec un héritage, l'héritage social et culturel, la tradition vivante, la culture transmise et apprise. Il y a une part de choix assurément dans la bretonnité. Mais n'importe qui ne peut certainement pas par simple stratégie personnelle identitaire se déclarer Breton du jour au lendemain, sans avoir aucun lien autre que tout à fait superficiel et ludique, avec la Bretagne. La bretonnité 
- comme n'importe quelle autre ethnicité - ne se réduit pas à un sentiment d'appartenance qui ne reposerait sur rien.

La notion d'ethnicité, mieux que celle d'identité, permet justement, me semble-t-il, d'inclure en même temps les aspects objectifs ou du moins relativement objectivables de la bretonnité - ce que l'on peut appeler la bretonnité en soi (les traits, les attributs qui singularisent les Bretons, leur appartiennent en propre et les différencient des autres) - et les aspects subjectifs - la bretonnité pour soi (la manière dont les Bretons se représentent, se sentent et s'identifient comme tels) et la bretonnité pour les autres et par les autres (les représentations que les autres ont des Bretons, les portraits, valorisants ou, plus souvent, du moins dans le passé, dévalorisants, que l'on a fait d'eux et qui ont plus ou moins été intériorisés par eux). Et cela permet aussi de ne pas faire l'impasse, d'introduire au contraire de manière explicite les aspects collectifs, combinés aux aspects individuels, de cette bretonnité. La bretonnité, c'est une affaire de représentations et de sentiments, d'identification, mais c'est aussi une affaire de vie en commun, au jour le jour, dans les choses et les activités de l'existence quotidienne.

En troisième lieu, il faut encore ajouter que l'ethnicité, ce n'est pas non plus la nationalité. De la nationalité, on peut dire que, désormais, sa signification est immédiatement et toujours politique : dans la nationalité il y a toujours, de fait, d'une manière ou d'une autre, la nation - soit une nation qui existe, soit qui a autrefois existé, soit qui existera et qui est alors en devenir, en projet -, la nation entendue au sens moderne du mot, la nation des nationalistes, c'est-à-dire une entité à la fois culturelle et politique, une formation sociale dotée d'un État, et pour laquelle, selon les termes de Gellner, les limites ethniques et les limites politiques doivent, par principe, coïncider.

L'ethnicité, telle qu'entendue ici, ne comporte pas en elle-même de dimension directement politique. Rien, autrement dit, dans l'ethnicité, aussi longtemps qu'elle n'est pas politiquement utilisée, manipulée, ne porte des gens qui se reconnaissent d'une même ethnicité à constituer une formation politique qui soit fondée sur cette communauté d'appartenance, dont cette ethnicité serait le principe. L'ethnicité peut, bien entendu, selon les situations, déboucher sur une telle revendication, se transformer alors en nationalité. Elle est même, comme cela a été dit, la voie royale vers la nationalité. Mais il n'y a rien là d'obligé. Une ethnicité peut parfaitement se vivre - les exemples sont nombreux, notamment (mais pas uniquement) dans les situations d'immigration - sans revendication de type national ou nationalitaire.

Cette bretonnité, maintenant, si l'on veut essayer de la mieux cerner, il convient en un premier temps de recenser et examiner les différents éléments susceptibles de la constituer, ses divers "marqueurs ", comme on dit. Et cela en partant non pas de l'importance plus ou moins grande qui pourrait être attribuée à ces éléments, ces composantes, ces traits d'ethnicité (ce qui serait dès le départ en préjuger), mais en allant du plus extérieur au plus interne. 
Soit depuis ce qui apparaît comme le mieux repérable, ce qui s'offre le plus immédiatement à l'observation, et qui semble apparemment le plus objectif ou du moins le mieux objectivable - apparemment car dès que l'on se livre à l'analyse, que l'on regarde les choses d'un peu près, ce n'est pas aussi simple que cela - jusqu'aux aspects les plus intimes et secrets, donc le plus subjectif. On est amené ainsi à passer en revue successivement les diverses composantes susceptibles de constituer la bretonnité.

L'apparence physique, d'abord, le phénotype (la fameuse tête de Breton ou de Bretonne que d'aucuns se flattent encore de reconnaître). Non que cela ait quelque chose à voir avec une race bretonne imaginaire. Mais il y a indiscutablement, et encore que cela soit bien difficile à saisir, une certaine manière commune à bien des Bretons et des Bretonnes d'utiliser leur corps, des techniques du corps, comme disait Mauss, un maintien, des postures, des mimiques, une gestuelle, etc., qui les distinguent parmi l'ensemble des Français - des gens du Midi notamment, mais aussi de bien d'autres.

À cette apparence physique a été lié intimement autrefois le costume qui après avoir été un très important marqueur, n'est plus aujourd'hui comme partout en Europe que folklorique.

Puis vient cet attribut d'identification qu'est le nom, le patronyme, le nom de famille, qui est toujours un élément parmi les plus forts, comme on sait, de l'identité individuelle (ne serait-ce que par la carte d'identité, le passeport, le chéquier, etc.) et peut l'être aussi de l'identité ethnique (ou nationale). Ce patronyme (auquel il faut éventuellement associer le prénom) peut être ou non à consonance bretonne, ce qui introduit des degrés dans la bretonnité vécue et perçue par les autres. Porter un nom immédiatement identifiable comme breton (Quéré, Madec, Le Cam, Le Bihan...), ce n'est pas la même chose que porter un nom d'allure française. Et cela réfère généralement à la distinction des Bas-Bretons et des Hauts-Bretons, de la Basse-Bretagne encore plus ou moins bretonnante et conservatoire de la bretonnité la plus " authentique " et de la Basse-Bretagne francisante.

Ce patronyme renvoie à l'origine familiale, à la filiation, à l'ascendance et à la descendance. Et là on est vraiment au cœur de la bretonnité-comme on le serait de toute ethnicité, qui se représente sur le mode généalogique, qui fonctionne à la perpétuation des aïeux : on est Breton d'abord parce que l'on est né de parents eux-mêmes Bretons et que l'on a été élevé par eux. Et l'on peut remonter de la sorte de génération en génération, dans la réalité des arbres généalogiques et, bien vite, par l'imagination et le mythe, aux origines mêmes du peuple breton, à nos ancêtres communs. Mais là aussi on retrouve les degrés de la bretonnité : n'avoir qu'une partie de ses ascendants bretons, cela éloigne du type généalogique idéal du Breton.

Origine aussi par le lieu : ce n'est pas la même chose d'être d'une ville ou de la campagne, de Basse Bretagne ou du Pays gallo, du Vannetais, du Trégor, de Cornouailles ou du Léon, etc. Ce qui renvoie également aux rap- 
ports, presque toujours de première importance en matière d'ethnicité et ils le sont ici tout particulièrement - au territoire et à ses paysages.

Et sont encore à considérer la langue, bien entendu, et les autres éléments de la culture, de même que la religion, les attitudes politiques... Pour terminer avec la psychologie collective, le caractère, le tempérament, sur lequel on a tant écrit et de manière si péremptoire, en des portraits négatifs, de dénigrement ou au contraire positifs : le Breton têtu, superstitieux, mélancolique, alcoolique, mauvaise tête mais quand même bon cœur, et le Breton fidèle, rêveur, imaginatif, idéaliste, à l'âme d'une exquise sensibilité, etc. Et enfin l'identité proprement dite, qui représente, ainsi que je l'entends, l'aspect proprement subjectif de l'ethnicité bretonne, de la bretonnité, l'identification à la Bretagne, sa culture, son histoire...

Recenser toutefois ces éléments, ces composantes, ces marqueurs de la bretonnité, pour nécessaire que cela soit, ne peut en aucune façon être suffisant. Aucun de ces éléments (pas même la langue, ou les langues si l'on admet que le gallo en est une), ni leur addition, la somme de ces composantes, ne permet de rendre vraiment compte de la bretonnité, ni de celle d'hier, encore moins de celle d'aujourd'hui. Cette bretonnité, cette ethnicité bretonne, s'est construite et continue de se construire - comme d'ailleurs toute ethnicité - dans des rapports sociaux-historiques, des relations et des interdépendances, qui sont ici des rapports entre majoritaires à minoritaires. Autrement dit entre dominants et dominés.

C'est-à-dire que la bretonnité ne peut se comprendre et s'analyser que de manière relationnelle et plus précisément par rapport à la situation minoritaire des Bretons dans l'ensemble français, leur situation de minorité. Ce qui signifie non pas tellement (comme on le comprend habituellement) le fait d'être moins nombreux, d'être en plus petit nombre, mais le fait d'être, à quelque degré, des mineurs sous tutelle et dépendance : mineurs comme le sont les enfants, jusqu'à leur majorité, mais aussi les vieillards frappés de sénilité (que l'on pense aux images si répandues de la "vieille Bretagne ", ses vieilles pierres, ses vieilles gens, ses vieilles mœurs et ses superstitions...), mineurs comme l'étaient autrefois les colonisés et aussi comme l'ont été longtemps, et le sont encore dans bien des sociétés, les femmes (et il n'est pas sans signification que les traits traditionnellement attribués aux Bretons, la sensibilité, la sentimentalité, la religiosité même, soient des traits habituellement associés à la féminité).

Situation de minoritaires qui a longtemps frappé les Bretons en tant que tels d'incapacité et de singularité dévalorisée, de différence infériorisée. Et qui a conduit à des phénomènes d'identité négative (honte de soi, haine de soi ou reniement de soi en tant que Breton, rejet d'une bretonnité méprisée, assimilée à l'archaïsme, au sous-développement, à la plouquerie). Et, en réaction à cette situation, au phénomène d'identité positive, manifeste aujourd'hui, mais qui vient en réalité d'assez loin, et qui se produit par retournement du stigmate en emblème, de ce qui était objet de honte en objet de fierté - processus tout à fait classique dans ce genre de situations, 
il suffit de penser aux discours sur la négritude et au slogan "black is beautiful ". La construction de cette identité positive - par valorisation de la langue, de la personnalité culturelle, de l'histoire, l'invention d'une néo-bretonnité - a été l'œuvre depuis le XIX ${ }^{\mathrm{e}}$ siècle de ce que l'on appelle, en prenant le terme dans un sens extensif, le mouvement breton, considéré dans sa branche culturelle surtout, toujours plus vivace et à l'action à long terme autrement profonde que sa branche politique.

Ce mouvement breton, l'Emsav, est, en effet, et a toujours été au cœur du problème breton et, aujourd'hui, de la bretonnité elle-même. Car ce qui apparaît assez clairement à l'analyse, c'est que si peut s'affirmer aujourd'hui une "identité " régionale et que se manifeste une néo-bretonnité d'une certaine vigueur, cela est, sur l'assez longue durée, l'œuvre du mouvement breton - quelles que soient ses grandes faiblesses (qui ne sont pas seulement numériques) et son histoire tourmentée, comme par ailleurs l'opinion, positive ou négative, favorable ou défavorable, que l'on en peut avoir. C'est ce mouvement breton, dans ses diverses composantes et à travers bien des vicissitudes, qui a joué dans le processus d'affirmation régionale le rôle primordial. Il faut ici renverser la perspective (qui est, généralement, et c'est bien normal, celle des acteurs de ce mouvement) : ce n'est pas la Bretagne, la "nationalité bretonne " ou le "peuple breton " (pas plus, d'ailleurs, que la soi-disant " situation coloniale " ou une " crise des structures économiques ", etc.) qui par un mystérieux processus mécanique ou organique ont produit le mouvement breton et ses revendications que l'on dit identitaires. C'est au contraire ce mouvement même qui a produit et d'une certaine manière inventé la Bretagne et la bretonnité tels que nous les connaissons à l'heure actuelle. Pas à partir de rien, bien entendu, en s'appuyant sur certaines réalités, en se fondant en tout premier lieu sur la maintenance passive au long des siècles de leur bretonnité par les paysans de Basse-Bretagne. Il n'en reste pas moins que c'est ce mouvement - analogue à beaucoup d'autres qui se sont constitués depuis le XIX siècle en Europe et ensuite ailleurs, sous la forme de régionalismes, de nationalismes et de nationalitarismes - c'est ce mouvement qui en donnant un sens, en quelque sorte, à ces réalités, à la personnalité culturelle bretonne, à l'histoire et à l'identité, a fait réellement exister la bretonnité.

On peut dire ainsi que cette Bretagne moderne et la néo-bretonnité ont été historiquement produites par l'idéologie du mouvement breton et son discours performatif, entendant par là une parole qui tend à faire surgir à l'existence ce qu'elle énonce ${ }^{2}$. L'énoncé performatif, dans celui du moins qui a acquis la crédibilité et la légitimité qui lui permet de réussir, c'est le verbe créateur, la parole qui se fait action, qui tend à produire les choses par les mots, à faire advenir à l'existence une réalité, en ce qu'il faut considérer comme un acte de véritable magie sociale. Une magie sociale qui

2. BouRDIEU, Pierre, "L'identité et sa représentation. Éléments pour une réflexion critique de l'idée de région ", Actes de la Recherche en Sciences Sociales, n 35, nov. 1980 et Ce que parler veut dire, Paris, Fayard, 1982. 
peut être, en certaines situations, parfaitement efficace. Agir par le langage sur les représentations du monde social, sur les images que les acteurs sociaux s'en font, c'est agir sur les réalités de ce monde. Le discours sur les réalités sociales est ainsi, dans certaines conditions, un élément de création, de construction et de transformation des réalités sociales et historiques.

Tout dépend ici, en plus évidemment de circonstances favorables, du pouvoir effectif de ceux qui le tiennent de faire reconnaître à leur discours l'effet qu'il entend produire. C'est-à-dire de leur capacité à produire à l'existence la chose nommée, leur pouvoir d'agir sur les choses par les mots. Et il faut bien admettre à cet égard que le mouvement breton n'a jamais jusqu'à ce jour rencontré un mouvement social de suffisante ampleur pour que son action puisse véritablement peser sur l'histoire - comme il en a été en d'autres contextes pour d'autres mouvements analogues, tenant sur telle ou telle autre collectivité humaine un discours similaire : au Québec, en Catalogne, au Pays basque, voire en Écosse... Ses idées, néanmoins, les représentations qu'il a élaborées de la Bretagne, de son histoire, de sa langue, de la personnalité culturelle, de l'identité bretonnes, et les expressions qu'il en a données (il suffit de penser, notamment, au drapeau, le gwenn-ha-du, et aussi bien sûr aux formes musicales et à la réinvention des festou noz), se sont finalement, depuis les années soixante, diffusées bien au-delà du noyau des militants de la cause bretonne, par une sorte de phénomène que l'on peut appeler de capillarité sociale. En ce sens l'idéologie de ce mouvement a commencé à avoir une certaine action sur la réalité. Et s'il est vrai que, comme le disait Glenmor, toute bonne graine honore le semeur, cela devrait conduire à prendre maintenant, sans complaisance mais non plus cette hostilité systématique qui interdit la compréhension, la juste mesure de ce mouvement dans l'histoire contemporaine de la Bretagne, et, de toute manière, de sa contribution décisive à ce qui apparaît comme le renouveau de la bretonnité.

Maintenant, que la montée de ce que l'on appelle les identités, de toutes sortes, recèle des dangers, il faudrait être aveugle et sourd pour le nier. Pour ce qui est du cas de la France, il n'est guère discutable que le communautarisme à l'américaine (ou peut-être plutôt l'idée que l'on s'en fait) soit une solution inadaptée à son histoire politique, aux valeurs généralement partagées depuis la consolidation de la République et de la démocratie. Il n'est certainement pas souhaitable d'aller à l'encontre de la tradition républicaine. Mais encore faut-il s'entendre sur celle-ci : de quelle tradition parle-t-on? De quelle République et de quelle Révolution se réclame-t-on? Est-ce celle des centralistes jacobins et bonapartistes, du Comité de Salut Public de 93 et sa centralisation renouant avec la politique constante de la monarchie d'Ancien Régime que Napoléon après le 18 brumaire parachèvera? Ou est-ce du printemps de la Révolution, de la Constituante, de la Déclaration des Droits de l'Homme et du citoyen, de la Fête de la Fédération de 1790, de ce moment de générosité et de grandeur, comme disait Tocqueville, " où les Français furent assez fiers de leur 
cause et d'eux-mêmes pour croire qu'ils pouvaient être égaux dans la liberté ${ }^{3}$ "?

Demander aujourd'hui plus de régionalisation, c'est s'inscrire dans la tradition du printemps de la Révolution, avant sa glaciation à l'heure de la Terreur et sa trahison par Bonaparte. Et se réclamer des principes les plus fondamentaux de la République. C'est s'inscrire dans un élargissement de la démocratie, par le bas, comme le serait, par le haut, la fédéralisation de l'Europe. On doit d'ailleurs se souvenir que c'est à cette première Révolution que les Bretons avaient, pour la plupart avec enthousiasme, adhéré. Le retournement, avec la Chouannerie, fut une réaction à ce qui apparaissait comme une confiscation de la Révolution par les Parisiens. Ce sont les Jacobins qui à l'époque ont jeté une partie des Bretons dans les bras de la Contre-Révolution.

Parler aujourd'hui d'élargissement de la démocratie, c'est la vouloir davantage pluraliste. Ce qui peut être considéré, dans une autre expression de la tradition républicaine française en ce qu'elle a de meilleur, comme un traitement plus large de la laïcité. Comme celle-ci a permis que soient reconnues les autres religions que la religion majoritaire, de même pourraient pleinement être reconnues dans une laïcité plus authentique d'autres cultures, d'autres ethnicités que la culture, l'ethnicité française. Et reconnue, notamment, la bretonnité. Breton, Français, Européen et citoyen du monde : c'est seulement, comme le disait aux lendemains de mai 1968 Morvan Lebesque, pour les nationalistes et les ennemis, cachés ou non, de la démocratie, qu'il y a incompatibilité entre ces appartenances et ces identités.

3. «Il y a eu, dans la révolution française, écrivait-il, deux mouvements en sens contraire qu'il ne faut pas confondre : l'un favorable à la liberté, l'autre favorable au despotisme. [...] La révolution [...] a été tout à la fois républicaine et centralisante. Ce double caractère de la révolution française est un fait dont les amis du pouvoir absolu se sont emparés avec grand soin. Lorsque vous les voyez défendre la centralisation administrative, vous croyez qu'ils travaillent en faveur du despotisme? Nullement, ils défendent une des grandes conquêtes de la Révolution. De cette manière, on peut rester populaire et ennemi des droits du peuple; serviteur caché de la tyrannie et amant avoué de la liberté." 
